\title{
DEAR: A DEVICE AND ENERGY AWARE ROUTING PROTOCOL FOR MOBILE AD HOC NETWORKS
}

\author{
Arun Avudainayagam $\quad$ Yuguang Fang Wenjing Lou \\ Department of Electrical and Computer Engineering \\ University of Florida \\ Gainesville, FL 32601 \\ Email: \{arun@dsp,fang@ece,wjlou@\}.ufl.edu
}

\begin{abstract}
Energy efficient routing has been the subject of intensive study in recent years. Most work has been focused on residual battery power related cost metrics for a homogeneous ad hoc network where all the nodes are considered identical in terms of functioning and available resources. In this paper, we propose a Device and Energy Aware Routing protocol (DEAR) for a heterogeneous wireless ad hoc network with two different classes of nodes, externally-powered nodes and battery-powered nodes. We embed both the energy and the device awareness into the routing protocol so that externallypowered nodes would forward more traffic and perform more routing functions. The simulation results show that DEAR protocol achieves better system lifetime when compared to the conventional energy efficient routing protocols. ${ }^{l}$
\end{abstract}

Keywords-- energy efficient routing, wireless ad hoc networks, sensor networks, minimum energy networks, power aware metrics

\section{INTRODUCTION}

Ad hoc networks are dynamically formed, infrastructureless, wireless multi-hop networks. The nodes configure themselves into a network and co-operatively maintain network connectivity. An intermediate node is used to forward packets if two nodes wishing to communicate are not within transmitting range of each other. Hence each node acts as a router and a host. The nodes discover multihop routes to each other by exchanging topology information in the form of control messages. Routing in ad hoc networks has been extensively studied over the past few years [1], [2].

\footnotetext{
${ }^{1}$ This work was supported in part by Office of Naval Research Young Investigator Award under grant N000140210464 and Office of Naval Research under grant N000140210554.
}

Traditional routing protocols can be broadly classified into two groups: Source Initiated (Reactive/On Demand) and Table Driven (Proactive). In the source initiated protocols [3][4], the routes are not computed until needed. This introduces a route-computation delay for the packets. In cases where this latency is not acceptable, table driven protocols [5] are used. In these protocols, routes to all destinations are pre-computed and stored as routing tables. The nodes update their routing tables periodically by exchanging routing table update packets. These initial routing protocols are only concerned with maintaining network connectivity in a highly dynamic environment and mainly deal with issues like route discovery and route maintenance. The routing decision is based more on the position of the node in the topology thus have the tendency to burden certain nodes with forwarding packets.

Energy conservation is an important issue in ad hoc networks as nodes are usually battery powered. Even though a node may not have any message of its own to transmit, its battery is drained when it acts as a router and forwards packets for other nodes. Energy aware routing (EAR) protocols have been proposed in response to the energy conservation requirement. An early goal of EAR is to minimize the total energy consumed by the network. This problem has been addressed, as minimum energy problem, in [6][9][11]. The basic approach is to minimize the average energy consumed per packet or per unit flow. One serious drawback of this approach is that nodes will have a wide difference in energy consumption. Nodes on the minimum energy paths will quickly drain out while the other nodes remain intact. This will result an early death of some nodes.

In the scenario where nodes need to work collaboratively, another objective of EAR is proposed, which is to maximize the time taken by the first node/sensor to fail because it runs out of battery power. This time is known as the system lifetime [7]. Singh etc. [6] proposed a set of power aware metrics based on battery power consumption at nodes. These metrics can be easily incorporated into the 
existing routing protocols. One of the metrics, the minimum cost per packet metric, aims to maximize the life of all nodes in the network. The shortest-cost routing algorithms using this metric achieved significant reduction in cost/packet over shortest-hop routing. The mean time to node failure was also increased. Chang and Tassiulas [7] proposed an energy conserving routing protocol to maximize the system lifetime by balancing the energy consumption among the nodes in proportion to their energy reserves. These proposed schemes embedded the energy awareness into the protocol and were proposed for a homogenous ad hoc network, where all the nodes are treated identical in terms of functioning and available resources. In addition, those schemes are suitable for static networks because the benefits come from the even distribution of traffic among different nodes. When the nodes are moving independently, the savings provided by these algorithms, if any, is negligible because of the difficulty of real-time re-configuration.

In this paper we propose a Device and Energy Aware Routing (DEAR) protocol for a heterogeneous ad hoc network. There exist two different classes of nodes, externally-powered nodes and the battery-powered nodes, in a heterogeneous ad hoc network. Besides the energy awareness, the proposed DEAR protocol also embeds additional information about the node type. We will show that, with both energy and device awareness, the system lifetime would be further increased by taking advantage of the extra capability and resources of externally-powered nodes while at the same time balancing traffic among the battery-powered nodes. This lifetime increment is achievable even when the nodes move independently with respect to each other.

This paper is organized as follows: In section 2 we first study the performance of the energy aware routing protocol and compare its performance with some conventional ad hoc routing protocols. In section 3 we introduce the device awareness in DEAR protocol, describe the design and operation of the routing protocol, and compare its performance with the protocol studied in section 2 . The paper is concluded in section 4 .

\section{ENERGY AWARE ROUTING PROTOCOL}

Power-aware metrics for determining routes of various objectives have been proposed in [6]. One metric that aims to maximize the life of all nodes in the network is defined as follows:

$$
c_{j}=\sum_{i=1}^{k-1} f_{i}\left(x_{i}\right)
$$

where $c_{j}$ is the cost of sending packet $j$ from node $n_{l}$ to node $n_{k}$ via intermediate nodes $n_{2} \ldots n_{k-1} ; x_{i}$ represents the total energy expended by node $i$ so far and $f_{i}\left(x_{i}\right)$ denotes the cost or weight of node $i$. Since $f_{i}$ represents a node's reluctance to forward packets, we have chosen

$$
f_{i}(t)=1 /\left(E_{i}-x_{i}(t)\right)
$$

where $f_{i}(t)$ denotes the cost of using node $i$ at time $t$; $x_{i}(t)$ denotes the energy expended by node $i$ until time $t$, and $E_{i}$ is the initial energy of node $i$ when the network is deployed. Thus, $f_{i}$ is the reciprocal of the residual energy of node $i$. Therefore, as the energy of a node decreases the cost of using that node increases.

The authors in [6] presented some results using this metric in a shortest path routing algorithm. However, they did not implement it as a protocol. Following their lead, we incorporate this metric as the link cost function into the Distributed Bellman-Ford (DBF) routing protocol for ad hoc networks. This implementation will be referred to as the EAR (Energy Aware Routing) protocol for the rest of this paper (not to be confused with the eavesdrop and register concept in sensor networks). One reason that we choose DBF is because the benefit of EAR protocol comes from the dynamic load balancing among different nodes. The protocol needs to keep track of the changing of the link costs in a timely fashion. The table driven protocol could gather these information actively while source initiated routing protocol presents a large lag in obtaining such changes. In order to reduce the routing overhead generated by the table driven protocol, our implementation of DBF just sends periodic updates of the routing table and does not trigger an update whenever there is a change in the routing table.

Whenever multiple paths exist from a source to a destination, EAR makes use of different paths and tends to balance the load by optimizing the residual energy. Hence, the system lifetime will be more than that obtained by using conventional routing protocols. We evaluate its performance and compare it with conventional ad hoc routing protocols by simulation. All the simulations are implemented within the GloMoSim library [12], which is a scalable simulation environment for wireless network systems.

Figure 1 shows the variation in system lifetime as a function of the number of nodes in the network for different routing protocols. The system lifetime decreases with an increase in the number of the nodes because the number of messages that a node might forward as a router 


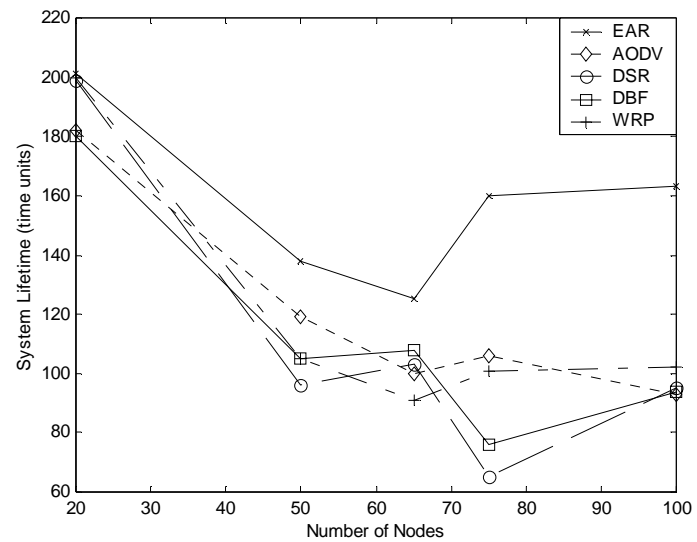

Figure 1. System Lifetime as a function of the number of nodes

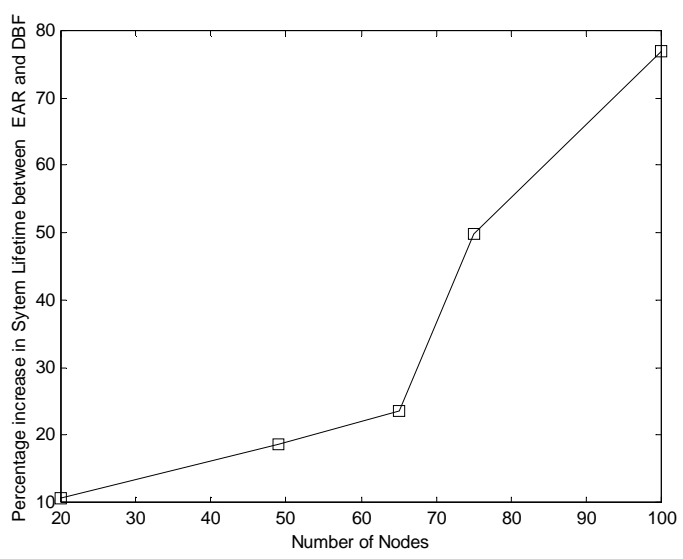

Figure 2. Percentage Increase in System Lifetime between EAR and DBF as a function of the number of nodes

increases. Figure 2 shows the percentage increase in system lifetime between EAR and DBF as a function of the number of nodes. As the number of nodes increase, it is more likely that each node has more routes (to every other node) to choose from. Since the load is balanced among a greater number of nodes, the percentage increase between EAR and DBF also increases.

Figure 3 shows the variation in system lifetime as a function of the edge density (network connectivity). The only way of increasing the network connectivity in wireless networks (keeping the number of nodes constant) is by bringing more nodes within transmitting range of each other. Thus, as the edge density increases, the number of direct transmissions from source to destination increases. Hence, as the connectivity increases, the shortest-cost path starts converging to the minimum-hop path. When $100 \%$ connectivity (edge density $=1$ ) has been reached, the network reduces to a single-hop network from

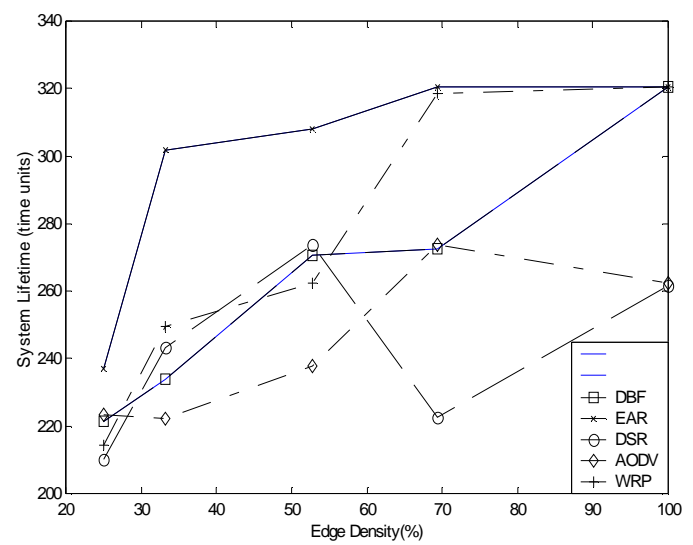

Figure 3. System Lifetime as a function of Edge Density

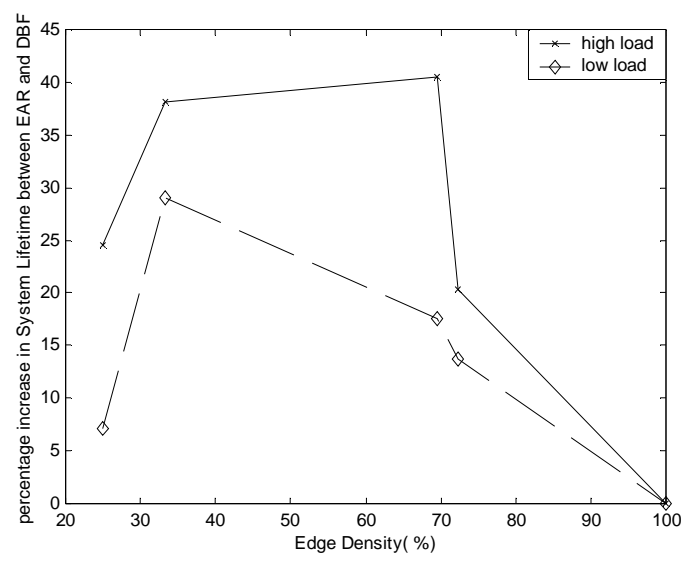

Figure 4. Percentage Increase in System Lifetime between EAR and DBF as a function of the edge density

a multi-hop network. Hence, the percentage increase in system lifetime is zero at $100 \%$ connectivity. This variation in percentage increase in system lifetime is shown in figure 4 for two different values of load. At a greater load, the percentage savings is greater because the cost differential between paths is greater at higher load than at a lower load and hence alternate routes are used more often.

We have not considered mobility of the nodes so far. All the above results hold true only for a static ad-hoc network (a sensor network kind of scenario). In [6], the authors have noticed that when the nodes move independently with respect to one another, then the savings obtained by using energy aware metrics will be small or even zero. This is because when the nodes are mobile, it is not necessary that there will be multiple paths from a source to a destination at all times. Hence, most often the shortest path will be the same as the minimum-hop path leading to zero savings. In 
the next section we present our DEAR protocol, which provides savings even when the nodes are independently moving.

\section{THE DEAR PROTOCOL}

\section{A. Motivation}

In conventional ad hoc network literature, the devices/nodes have been assumed to be identical in terms of available resources and the roles they played as a router. However, recently there has been a paradigm shift. Many researchers investigate heterogeneous ad hoc networks with the nodes not necessarily identical to each other [10]. We observe that in practical ad hoc network deployment there are some nodes that are powered by a source that is not critically limited in energy. For example, consider the deployment of an ad hoc network in a disaster situation, where the handsets carried by the rescue workers are the nodes. Then, there might be a case where a rescue worker plugs his handset in a charger in an automobile/ambulance. In a military application, some soldiers may be in jeeps or tanks that have chargers in them. In a commercial deployment, a user may be in a car with a charger. In such scenarios, these devices are not limited in power in the sense that their power reserves are very large compared to the battery power of other nodes. The DEAR protocol that we propose exploits this fact and makes use of device awareness to enhance the routing.

\section{B. Design and Operation of DEAR protocol}

DEAR stands for Device and Energy Aware Routing. A node is said to be device aware if it can distinguish between two states: it is powered by its internal battery or it is powered by an external source. We assume that the cost of using a node powered by an external source as zero. Thus we incorporate a redirect scheme in DEAR that actively redirects the packets to the powered nodes for power saving operations. We also assume that an external powered node has the capability to increase its transmission power to a higher level so that it can reach any other node in the network in one hop.

The device aware redirect scheme is designed as follows. A conventional routing table entry should include at least the following fields: destinationAddr, cost, nextHop. An additional binary field, deviceType, is added to this structure for device awareness. A 0 indicates the node destinationAddr running on its own battery and a 1 indicates an externally-powered node. Each node maintains a routing table and an additional redirect table. The redirect table entry has the following structure: destinationAddr, redirectToAddr, indicating where
TABLE UPDATE ALGORITHM

on receiving routing table update from neighbor:

UpdateRoutingTable( ); /*identical to DBF update procedure*/

/*find the least cost powered node and the cost $*$ /

ShortestCostToPoweredNode=infinite;

for each entry $d$ in the routing table (RT), do \{

if (RT[d].deviceType $==1 / *$ device is powered node ${ }^{*}$

AND

RT[d].cost < ShortestCostToPoweredNode) \{

ShortestCostToPoweredNode = RT [d].cost;

\}

RedirectNode $=\mathrm{RT}[\mathrm{d}]$.destinationAddr;

\}

/*Update redirect table*/

for each entry $d$ in routing table(RT) and redirect table(RD), do \{

if (RT[d].cost < ShortestCostToPoweredNode)

$\mathrm{RD}[\mathrm{d}]$.redirectToAddr $=$ RedirectNode; else

$\mathrm{RD}[\mathrm{d}]$.redirectToAddr $=-1$;

\}

Figure 5 Routing and Redirect Table Update Algorithm

(redirectToAddr) the packet leaving the current node to destination destinationAddr should be redirected. A -1 in field redirectToAddr indicates no redirection is needed. Whenever a routing table update is received, it updates its routing table as done in EAR or DBF. After updating its routing table, the node browses through its routing table and determines the shortest cost to reach any externally powered device. Let $C$ represent this cost and let $P$ be the id of the corresponding device. The redirect table will be updated as summarized in Figure 5. That is, for each entry in the redirect table, if the cost to that destination is bigger than $C$, packets to that destination will be redirect to $P$, correspondingly the field redirectToAddr will be set to $P$. Otherwise, the field redirectToAddr will be set to -1 , indicating that packets to that destination should follow the routing table, but not be redirected.

Whenever a node gets a packet to be forwarded, it gets the destination address from the header and it looks at the corresponding entry in the redirect table. If the entry in the redirect table is -1 , the node just forwards the packet to the nextHop according to the routing table for that particular destination. If the entry in the redirect table is the id of some other node, it redirects the packet to that particular node.

Once a powered node receives a packet, it checks if the destination of the packet is one of its neighbors (single hop nodes). If so, the node unicasts it to that particular 


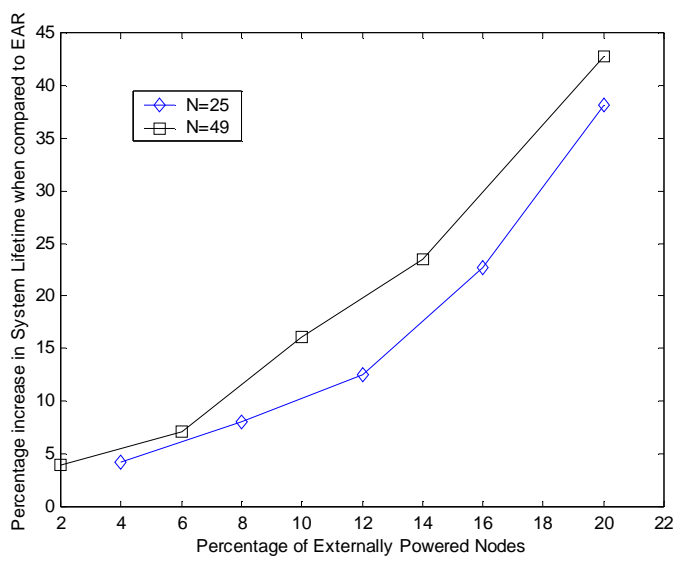

Figure 6. Percentage increase in system lifetime between DEAR and EAR as a function of the number of powered nodes

destination. If not, it boosts its transmit power to cover the entire network and unicasts the packet to its destination. So it is just a single hop from a powered node to the destination. Since the node is externally powered it can boost its transmit power to cover any distance. The amount by which the power has to be increased can be handled in different ways. If the area of deployment is known then the amount by which the transmit power should be increased to cover the entire area can be pre-programmed into the node. Else, if the nodes also exchange hop count information (as in DBF) apart from the cost information, then the externally powered node will know the number of hops to the intended destination and can correspondingly increase the transmit power. If the nodes had different transmit powers, DEAR operation could be combined with a protocol that exchanges transmit power information like PARO [10] to exactly determine the amount of transmit power required to reach the destination. This, however, would involve a little more over-head. For our simulations, we pre-programmed the increase in transmit power required to cover the area of network deployment into the protocol. In practice, even the powered node has a limitation in transmitted power, therefore, a powered node can increase the transmit power level to the maximum or until another powered node is found. This approach will be investigated later.

\section{Performance}

Since the cost of reaching a powered node was lower, a savings in energy (a number of hops are avoided) is obtained. Figure 6 shows the percentage improvement in system lifetime between DEAR and EAR as a function of the number of powered nodes. It is observed that the system lifetime increases as the number of powered nodes increases, which is expected. We would like to state that

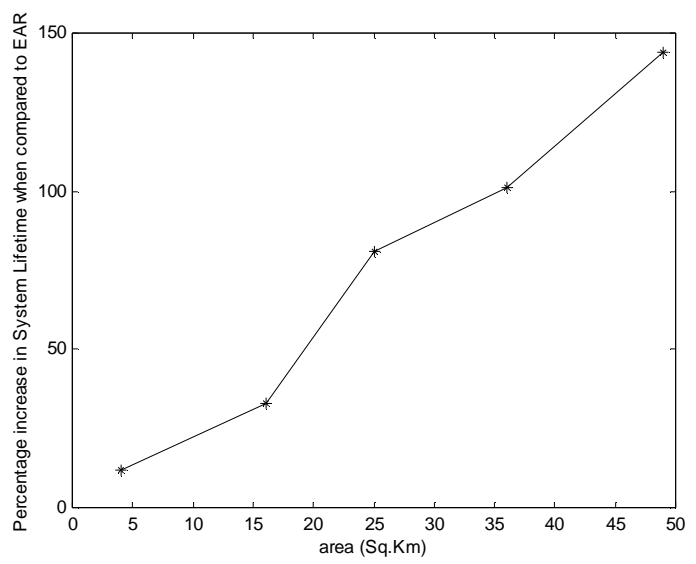

Figure 7. Percentage Increase in system lifetime between DEAR and EAR as a function of the area of deployment

mobility model developed by Johnson and Maltz [3] was used for our simulation. If any savings is to be expected from EAR, there should be multiple routes between pairs of nodes. In a mobile environment, the only way to ensure multiple routes is by reducing the area over which the nodes move. While this only makes it more likely for EAR to provide savings, it does not guarantee better performance. This is because when the area of deployment is reduced, it is likely that the connectivity increases and as mentioned in section 2, savings might decrease. However, in applications of mobile ad hoc networks, this constraint on the area of deployment is not practical. Hence as the area increases, the savings obtained by EAR if any, will actually decrease. It is also expected that as the area increases the number of hops between two nodes will increase. However, if we use DEAR, as the area increases, if a route exists with a smaller cost to a powered device, then the savings will be greater because it is more likely that we will avoid a greater number of hops. Hence with an increase in area, the percentage increase in system lifetime when compared to EAR should actually increase which is observed in figure 7 .

\footnotetext{
${ }^{2}$ Note: In our simulations a modified version of the MACA[13] protocol was used in the MAC layer. Whenever a powered node has to boost the transmit power and deliver a message, it transmits a special RTS packet on a separate control channel. Any node receiving the special RTS packet stops its transmissions, if any. This prevents collisions with parts of the ad hoc network that are not "visible" to the powered node. This pre-emptive MAC protocol is not optimal and better protocols will be investigated in the future. However, for low bit rate applications, the penalty of using this MAC protocol may not be severe.
} 


\section{CONCLUSIONS}

In this paper, we compare the performance of a simple Energy Aware Routing protocol with a few conventional protocols using the system lifetime as the performance measure. A limitation of the application of this protocol to mobile networks is identified. The crux of our paper is not to propose better energy aware metrics but to show how these metrics can be adapted to mobile ad hoc networks. We introduce the DEAR protocol and show how embedding some device awareness into a traditional energy aware routing protocol could be used to significantly increase the system lifetime and could be used to adapt energy aware routing protocols into the mobile environment. We would like to state that device awareness could be embedded in any protocol at any layer to improve the system lifetime.

\section{REFERENCES}

[1] D.B. Johnson. "Routing in Ad Hoc Networks of Mobile Hosts". Proceedings of the IEEE Workshop on Mobile Computing Systems and Applications, Dec 1994.

[2] J. Broch, D.A. Maltz, D.B. Johnson, Y.C Hu, J. Jetchva. "A Performance Comparison of Multi-Hop Wireless Ad Hoc Network Routing Protocols". Proceedings of the Fourth Annual ACM/IEEE International Conference on Mobile Computing and Networking (MobiCom '98).

[3] D.B. Johnson and D.A. Maltz. "Dynamic Source Routing in Ad Hoc Wireless Networks". In Mobile Computing, Edited by Tomasz Imielinski and Hank Korth, Chapter 5. Kluwer Academic Publishers 1996.

[4] C.E. Perkins, E.Royer. "Ad Hoc On Demand Distance Vector (AODV) Routing". Proceedings of the Second IEEE Workshop on Mobile Computing Systems and Applications, Feb 1999.
[5] C. E. Perkins and P. Bhagawat. "Highly Dynamic Destination-Sequenced Distance-Vector Routing (DSDV) for Mobile Computers". Proceedings of the SIGCOMM '94 Conference on Communications Architectures, Protocols and Applications, Aug 1994.

[6] S. Singh, M. Woo and C.S. Raghavendra. "Power Aware Routing in Mobile Ad Hoc Networks". Proceedings of the Fourth Annual ACM/IEEE International Conference on Mobile Computing and Networking (MobiCom '98).

[7] J. Chang and L. Tassiulas. "Energy Conserving Routing in Wireless Ad Hoc Networks." Proceedings INFOCOM 2000, Mar 2000.

[8] W. Heinzelman, A. Chandrasekaran and $\mathrm{H}$. Balakrishnan. "Energy Efficient Communication Protocol for Wireless Micro Sensor Networks". Proceedings of the $33^{\text {rd }}$ Hawaii International Conference on System Sciences (HICSS '00), Jan 2000.

[9] V. Rodoplu and T.H. Meng. "Minimum Energy Mobile Wireless Networks". Proceedings of 1998 IEEE International Conference on Communications (ICC '98), Jun 1998.

[10] J. Gomez, A. T. Campbell, M. Naghshineh, and C. Bisdikian. "Power-Aware Routing in Wireless Packet Networks". Proceedings of Sixth IEEE International Workshop on Mobile Multimedia Communications, San Diego, CA, Nov 1999.

[11] C.-K.Toh. "Maximum Battery Life Routing to Support Ubiquitous Mobile Computing in Wireless Ad Hoc Networks ". IEEE Communications Magazine, Vol.39 Issue 6, June 2000.

[12] M. Takai, L. Bajaj, R.Ahuja, R. Bagrodia, M. Gerla, GloMoSim: a scalable network simulation environment, Technical Report 990027, UCLA, Computer science department, 1999.

[13] P.Karn, "MACA-A new channel access method for packet radio", in ARRL/CRRL Amateur Radio $9^{\text {th }}$ Computer Networking Conference, pp-134-140, ARRL,1990. 